\title{
Lo mejor del Congreso Uruguayo de Cardiología 2020
}

\author{
Dres. Carlos Guamán'1, Estefanía de la Fuente², Keril Gianoni², David de Sosa
}

\section{Resumen}

El 37. ${ }^{\circ}$ Congreso Uruguayo de Cardiología se llevó a cabo exclusivamente en modalidad virtual, del 26 al 28 de mayo. Contó con invitados nacionales e internacionales, permitiendo el desarrollo de un espacio de actualización en distintas áreas de la cardiología. Al igual que en años anteriores, se destinó un espacio a la actividad científica efectuada por investigadores nacionales mediante la presentación de temas libres. Durante 2020 se presentaron diversos trabajos en este formato que fueron sometidos a un proceso de revisión y aprobación por el comité científico, cuya presentación fue diferida por la pandemia por COVID-19. A continuación, comentamos algunos de los trabajos presentados en el congreso, a saber:

- Implante primario de marcapaso definitivo cardíaco en población pediátrica y evolución: experiencia de 15 años en centro de referencia cardiológico pediátrico en Uruguay.

- Calidad de la anticoagulación oral con warfarina en una policlínica de cardiología, seguimiento de un año.

- Valor pronóstico de la anemia leve/moderada y/o la ferropenia preoperatoria en el posoperatorio de cirugía cardíaca.

- Impacto pronóstico del ecocardiograma de estrés con ejercicio físico negativo en una unidad de dolor torácico. Período 2019-2020 con seguimiento a un año.

- Estrategia invasivoconservadora en el infarto con elevación del ST. Prueba de concepto.

- Impacto cardiovascular de la pandemia por COVID-19 durante el primer semestre de 2020 en Uruguay. Estudio ecológico.

- Correlación entre parámetros hemodinámicos y captación de ${ }^{18} \mathrm{~F}$-fluoruro mediante PET a corto plazo luego de sustitución valvular aórtica.

- Indicación, resultados y mortalidad de la angioplastia coronaria con implante de stent en tronco de arteria coronaria izquierda no protegido.

Palabras clave: MARCAPASO

ANTICOAGULACIÓN

CIRUGÍA CARDÍACA

ECOCARDIOGRAMA

ANGIOPLASTIA

\section{The best of the 2020 Uruguayan Congress of Cardiology \\ Summary}

The $37^{\text {th }}$ Uruguayan Congress of Cardiology was held in virtual mode, from May 26 to 28. It had national and international guests, allowing the development of an update space in different areas of cardiology. As in previous years, a space was set aside to present the scientific activity carried out by national researchers through the presentation of free topics. Various free topics of 2020 were reviewed and approved by the scientific committee, whose presentation was deferred due to the COVID-19 pandemic. Below we comment on some of the papers presented at the congress, as follows:

1. Centro Cardiovascular Universitario, Hospital de Clínicas. Montevideo, Uruguay.

2. Centro Cardiovascular Casa de Galicia. Montevideo, Uruguay.

Correspondencia: Dr. Carlos Guamán. Correo electrónico: cgv0792@gmail.com

Los autores declaran no tener conflictos de intereses.

Recibido Oct 15, 2021; aceptado Oct 27, 2021 
- Primary implantation of a definitive cardiac pacemaker in the pediatric population and evolution: 15 years of experience in a pediatric cardiology referral center in Uruguay.

- Quality of oral anticoagulation with warfarin in a cardiology polyclinic, one-year follow-up.

- Prognostic value of mild/moderate anemia and/or preoperative iron deficiency in the postoperative period of cardiac surgery.

- Prognostic impact of stress echocardiography with negative physical exercise in a chest pain unit. 2019-2020 period with one-year follow-up.

- Invasiveconservative strategy in ST elevation infarction. Proof of concept.

- Cardiovascular impact of the COVID-19 pandemic during the first half of 2020 in Uruguay. Ecological study.

- Correlation between hemodynamic parameters and 18 F-fluoride uptakes by PET in the short term after aortic valve replacement.

- Indication, results and mortality of coronary angioplasty with unprotected left main coronary artery stent implantation.

Key words: $\quad$ PACEMAKER

ANTICOAGULATION

HEART SURGERY

ECHOCARDIOGRAM

ANGIOPLASTY

\section{O melhor do Congresso Uruguaio de Cardiologia 2020}

\section{Resumo}

O 37. ${ }^{\circ}$ Congresso Uruguaio de Cardiologia foi realizado de forma virtual, de 26 a 28 de maio. Contou com convidados nacionais e internacionais, permitindo o desenvolvimento de um espaço de atualização nas diferentes áreas da cardiologia. À semelhança dos anos anteriores, foi reservado um espaço para a apresentação da atividade científica desenvolvida por investigadores nacionais através da apresentação de temas livres. Foram apresentados diversos temas livres revisados e selecionados pela comissão científica correspondente ao ano de 2020, cuja apresentação foi adiada devido à pandemia do COVID-19. A seguir comentamos alguns dos trabalhos apresentados no congresso.

- Implante primário de marcapasso cardíaco definitivo na população pediátrica e evolução: 15 anos de experiência em centro de referência em cardiologia pediátrica no Uruguai.

- Qualidade da anticoagulação oral com varfarina em policlínica de cardiologia, seguimento de um ano.

- Valor prognóstico de anemia leve/moderada e/ou deficiência de ferro pré-operatória no pós-operatório de cirurgia cardíaca.

- Impacto prognóstico da ecocardiografia sob estresse com exercício físico negativo em uma unidade de dor torácica. Período 2019-2020 com acompanhamento de um ano.

- Estratégia invasivoconservadora no infarto com supra desnivelamento de ST. Prova de conceito.

- Impacto cardiovascular da pandemia COVID-19 durante o primeiro semestre de 2020 no Uruguai. Estudo ecológico.

- Correlação entre parâmetros hemodinâmicos e captação de fluoreto de $18 \mathrm{~F}$ por PET em curto prazo após a troca da válvula aórtica.

- Indicação, resultados e mortalidade de angioplastia coronária com implante de stent de tronco de coronária esquerda desprotegido.

Palavras chave: MARCAPASSO

ANTICOAGULAÇÃO

CIRURGIA CARDÍACA

ECOCARDIOGRAMA

ANGIOPLASTIA 


\section{Implante primario de marcapaso definitivo cardíaco en población pediátrica y evolución: experiencia de 15 años en centro de referencia cardiológico pediátrico en Uruguay}

El implante de marcapaso en la población pediátrica es infrecuente, correspondiendo a menos de $1 \%$ de los implantes totales. Cuando es requerido, es más común en menores de 10 años, en relación con la población adulta cuya edad media es de 75,5 años ${ }^{(1)}$. Aunque en los pacientes pediátricos la experiencia en el uso de marcapasos es relativamente escasa, los múltiples avances en la cirugía cardíaca infantil han propiciado un aumento en el número de implantes de marcapaso.

Respecto a la técnica en la edad pediátrica, históricamente se prefirió el implante epicárdico, debido no solo al tamaño de los generadores y a que los cables endovenosos no se consideraban apropiados, sino también a que muchas de las indicaciones de implante eran por lesiones secundarias a la corrección de alguna cardiopatía congénita, que en algunos casos no permitía el acceso endovenoso al ventrículo derecho. No debe dejarse de lado un factor no menor relacionado con el crecimiento y la estética, siendo peor el resultado cuanto más pequeño es el paciente ${ }^{(2)}$. En la actualidad, el implante de marcapaso endocárdico en esta población se utiliza cada vez más, por las ventajas que este abordaje ofrece, como la obtención de umbrales de captura más bajos y un menor riesgo de fractura de los electrodos ${ }^{(3)}$.

El Dr. Mateo Ríos y col. diseñaron un estudio observacional, retrospectivo, efectuado en un centro de referencia cardiológico pediátrico en Uruguay, en el cual se incluyeron pacientes menores de 18 años que requirieron un primer implante de marcapasos definitivo en un período de 15 años (2004-2019), excluyendo otros dispositivos. Su objetivo fue caracterizar la práctica realizada en el centro y analizar su evolución, tanto a corto como a mediano plazo. Consideraron variables demográficas, patología de base, dispositivos implanta- dos, controles y evolución ${ }^{(4)}$.

En el período de estudio se realizaron 1.427 procedimientos, de los cuales 98 fueron en pacientes menores de 18 años. Entre ellos, 53 cumplieron con los criterios de inclusión. Las características basales fueron: 53\% sexo femenino, edad promedio de 7,1 $\pm 5,3$ años, indicados $89 \%$ por bloqueo auriculoventricular completo (BAVC, $22 \%$ posoperatorios), $8 \%$ por disfunción sinusal y $4 \%$ por síndrome QT largo asociado a bradicardia.

El 55\% de los primoimplantes fueron endocárdicos y el 45\% epicárdicos. El modo de estimulación más utilizado fue VVI (58\%), seguido de DDD (26\%). Previo al alta se observaron complicaciones en 2 pacientes (4\%): un hematoma y una dehiscencia de la herida.

Se obtuvo información del seguimiento a $98 \pm 65$ meses (8,2 $\pm 5,4$ años). En ese período se registraron $9 \%$ de complicaciones (3 fallas de umbral, una de las cuales requirió reintervención, 1 exteriorización y 1 fallecimiento por mediastinitis). Existieron 7 fallecimientos, aunque solo uno se asoció estrictamente al implante realizado, y otro en el período inmediato posimplante relacionado con la patología de base.

Los autores concluyeron que los resultados obtenidos son acordes a los reportes internacionales sobre el tema, siendo un procedimiento seguro en el servicio, con baja incidencia de complicaciones asociadas al implante, no dejando de lado que la población estudiada tiene una alta mortalidad a largo plazo por sus patologías de base y la necesidad de numerosas reintervenciones. En series internacionales, las indicaciones de los implantes varían con respecto a esta experiencia nacional, con al menos 50\% de las indicaciones por BAVC posoperatorio, seguidas por el BAVC congénito y la disfunción sinusal ${ }^{(2)}$.

\section{Calidad de la anticoagulación oral con warfarina en una policlínica de cardiología, seguimiento de un año}

El tratamiento anticoagulante con inhibidores de la vitamina $\mathrm{K}$ como warfarina ha demostrado disminuir la incidencia de las complicaciones tromboembólicas en los pacientes con fibrilación auricular (FA). Sin embargo, por sus propiedades farmacocinéticas y farmacodinámicas, el punto exacto entre obtener su máxima eficacia (prevenir eventos tromboembólicos) y minimizar su riesgo (prevención de hemorragias) es difícil de alcan$\operatorname{zar}^{(5)}$. La eficacia y seguridad están relacionadas con el nivel de anticoagulación, el cual es valorado mediante el seguimiento del índice internacional normalizado y la determinación del tiempo en rango terapéutico (TTR). Mantener un adecuado nivel de anticoagulación es complejo, debido a la amplia variabilidad inter e intraindividual de su 
efecto, sus múltiples interacciones tanto farmacológicas como dietéticas y su estrecho rango terapéutico $^{(6)}$. Algunas revisiones recomiendan que el TTR óptimo para cualquier patología debe ser mayor a $65 \%-70 \%$, un nivel que permite reducir significativamente la incidencia de complicaciones tromboembólicas y hemorrágicas. No obstante, para cumplir con este objetivo, es necesario un sistema organizado de seguimiento, monitoreo, adecuada comunicación y educación del paciente y su entorno familiar ${ }^{(6,7)}$.

La Dra. Erika Mirandetti y col. diseñaron un estudio observacional, descriptivo, retrospectivo y analítico de pacientes anticoagulados con warfarina por al menos 1 año, procedentes de una policlínica de cardiología, clasificándolos en dos grupos: 1) pacientes con indicación por FA o flutter auricular y 2) portadores de prótesis valvular. Su objetivo fue valorar la calidad de la anticoagulación oral con warfarina, comparando el TTR entre ambos grupos, e identificar tanto los factores asociados a un adecuado nivel de anticoagulación como las complicaciones asociadas a la terapia (seguridad) $)^{(8)}$.

Se definió como nivel de anticoagulación "adecuado" a un TTR mayor a 65\%. En total se analizaron 270 pacientes, de los cuales 202 cumplieron los criterios de inclusión, siendo el 56,96\% de sexo femenino. En el grupo de FA o flutter auricular se incluyeron 137 pacientes, y en el grupo de prótesis valvular mecánica 65 pacientes. La media de TTR fue de 59,68 \pm 22 , sin encontrar una diferencia estadísticamente significativa entre ambos grupos.

Los autores realizaron un análisis mediante una regresión multivariada, en la cual evidenciaron diversas variables predictoras de mayor TTR: edad, educación terciaria incompleta y $\mathrm{CHA}_{2} \mathrm{DS}$ ${ }_{2}$ VASc de 7; por otra parte, de las variables asociadas con un menor TTR fueron: $\mathrm{CHA}_{2} \mathrm{DS}_{2}$ VASc de 1 , HASBLED elevado ( $\geq 3$ ), la ingesta de clopidogrel y la baja adherencia al tratamiento. En la evolución no hubo una alta incidencia de complicaciones: solo 4 pacientes presentaron hemorragias graves, 2 presentaron complicaciones trombóticas y 31 tuvieron hemorragias menores. No se demostraron variables asociadas al desarrollo de estas complicaciones.

La Dra. Mirandetti concluyó que la calidad de anticoagulación de esta población ambulatoria fue subóptima, con una baja incidencia de complicaciones, con variables relacionadas con un mejor nivel de anticoagulación como edad, educación terciaria incompleta y $\mathrm{CHA}_{2} \mathrm{DS}_{2} \mathrm{VASc}$ de 7, y otras con un peor nivel de anticoagulación como $\mathrm{CHA}_{2} \mathrm{DS}_{2}$ VASc de 1 , HASBLED alto y baja adherencia al tratamiento. Los autores proponen que estas variables podrían ser de utilidad para el desarrollo de protocolos de seguimiento más individualizados en la policlínica de anticoagulación.

\section{Valor pronóstico de la anemia leve/moderada y/o la ferropenia preoperatoria en el posoperatorio de cirugía cardíaca}

La anemia en los pacientes en preoperatorio de cirugía cardíaca es un hallazgo común. Varios estudios han encontrado que es un factor de riesgo importante, asociado con peores resultados como mayor mortalidad, morbilidad y hospitalización prolongada ${ }^{(9)}$. Mientras tanto, la ferropenia también es frecuente en el preoperatorio de cirugía cardíaca de indicación electiva, y ha demostrado aumentar los requerimientos de transfusiones y la fatiga en el posoperatorio ${ }^{(1)}$.

Con este problema en el horizonte, la Dra. Victoria Hernández y col. diseñaron un estudio ${ }^{(11)}$ de cohortes en dos centros nacionales, para evaluar el valor pronóstico tanto de la anemia leve a moderada como de la ferropenia en los pacientes cursando el preoperatorio de cirugía cardíaca. Incluyeron a todos los pacientes mayores de 18 años que fueron sometidos a cirugía cardíaca de coordinación durante un período de 6 meses, excluyendo a los pacientes con endocarditis infecciosa, síndrome aórtico agudo, patología de pericardio, enfermedad renal crónica en diálisis y anemia severa. Estudiaron 3 cohortes: 1) un grupo control: sin anemia ni ferropenia; 2) pacientes con ferropenia aislada y 3) pacientes con anemia leve a moderada.

Las autoras captaron una población de 167 pacientes, con una media de edad de 68 años, $y$ $61,68 \%$ hombres, encontrando una alta prevalencia de anemia leve/moderada y/o ferropenia, cuya presencia se asoció a una tendencia consistente de mayor riesgo de complicaciones posoperatorias e internaciones más prolongadas. 


\section{Impacto pronóstico del ecocardiograma de estrés con ejercicio físico negativo en una unidad de dolor torácico. Período 2019-2020 con seguimiento a un año}

El dolor torácico es una de las causas más frecuentes de consulta en emergencia. Las unidades de dolor torácico (UDT) llevan adelante protocolos que permiten la estratificación de riesgo de los pacientes, permitiendo así el alta con seguridad ${ }^{(12)}$. El empleo del ecocardiograma con estrés ergométrico (EEE) en dichos protocolos tiene valor agregado sobre la prueba ergométrica sin imagen.

Para conocer los resultados del empleo del EEE en contexto de UDT, el Dr. Ignacio Farro y col. diseñaron un estudio descriptivo, prospectivo, observacional y unicéntrico ${ }^{(13)}$. Los autores incluyeron todos los pacientes enviados a EEE en protocolo de UDT de bajo y moderado riesgo (analizado mediante el escore HEART), desde mayo de 2019 a diciembre de 2020. Se consideró como un EEE "positivo" a la presencia de nuevas alteraciones en la motilidad sectorial inducidas por el esfuerzo. Se evaluó la presencia o no de reserva contráctil y la respuesta diastólica. La aparición de eventos adversos (consulta por ángor, necesidad de cineangiocoronariografía y muerte) se confirmó mediante la revisión de las historias clínicas.

En el análisis se incluyeron 96 sujetos, con una edad media de 59,4 años, 34\% mujeres, excluyéndose dos pacientes por presentar alteraciones de la contractilidad basales. El 18\% de los EEE fueron positivos (41\% de alto riesgo, grupo 1: G1), 64\% negativos (grupo 2: G2) y 18\% insuficientes. No ocurrieron complicaciones durante el estudio. El 47\% de los pacientes con un escore HEART intermedio tuvieron un EEE negativo, mientras que el $8 \%$ de aquellos de bajo riesgo fueron reclasificados como de alto riesgo tras el EEE. Los investigadores pudieron realizar el seguimiento en el $100 \%$ del G1 y encontraron que el $97 \%$ de ellos no presentó eventos adversos durante un período de $12 \pm 6$ meses. Dos pacientes presentaron eventos adversos: recurrencia de dolor a los 12 meses (MIBI con isquemia leve y tratamiento médico) y recurrencia de dolor, angioplastia (ATC) de un ramo diagonal, sin otras lesiones coronarias significativas. Los autores concluyen que el EEE es una prueba segura que permite otorgar el alta en forma confiable, disminuyendo los tiempos de internación, y que una prueba negativa se asocia con un buen pronóstico a mediano plazo.

\section{Estrategia invasivoconservadora en el infarto con elevación del ST. Prueba de concepto}

En la actualidad existe evidencia de que la ATC primaria con implante de stent es el mejor tratamiento para los pacientes con infarto agudo de miocardio con elevación del segmento ST (IAMCEST). Algunos estudios han investigado el beneficio del aplazamiento del implante de stent en estos pacientes, para conservar la función de la microvasculatura y reducir su obstrucción ${ }^{(15)}$. El estudio DANAMI 3-DEFER, que incluyó 1.215 pacientes, demostró que el aplazamiento del implante de stent en el IAMCEST (ATC exclusiva con balón) se asoció con mayor necesidad de revascularización del vaso culpable ${ }^{(15)}$. La guía 2017 sobre el tratamiento del IAMCEST de la Sociedad Europea de Cardiología no recomienda el aplazamiento del implante de stent (clase de recomendación III) ${ }^{(14)}$.

En el congreso, el Dr. Juan Albistur y col. presentaron los resultados preliminares de un estudio prospectivo, analítico y controlado en curso $^{(16)}$.
Incluyeron 49 pacientes asistidos en un centro uruguayo por IAMCEST de menos de 12 horas de evolución, derivados para ATC primaria, excluyendo aquellos con disecciones coronarias, reestenosis de stent y revascularizados quirúrgicos. El objetivo fue evaluar la eficacia y la seguridad de la estrategia invasivoconservadora con ATC de balón/tromboaspiración y eventual implante diferido de stent, guiado por fisiología coronaria. La mediana de seguimiento fue de 174 días (94,5200 días). Nueve pacientes fueron derivados a la estrategia invasivoconservadora, destaca en este grupo que todos presentaron flujo de reperfusión TIMI 3, 2 requirieron implante de stent, y todos permanecían asintomáticos y sin eventos adversos hasta la fecha de la presentación.

Estos hallazgos son sugerentes de que, en cierta población seleccionada de pacientes con IAMCEST, la estrategia invasivoconservadora podría demostrar beneficios. 


\section{Impacto cardiovascular de la pandemia por COVID-19 durante el primer semestre de 2020 en Uruguay. Estudio ecológico}

A fines de diciembre de 2019 en Wuhan, China, se reportaron los primeros casos de COVID-19, patología que se propagó con rapidez. La Organización Mundial de la Salud la declaró emergencia de salud pública de importancia internacional el 30 de enero de 2020 $0^{(17,18)}$. En Uruguay, el 13 de marzo se identificaron lo primeros casos, e inmediatamente fueron implementadas medidas de distanciamiento social y cuarentena no obligatoria, obteniendo un control adecuado de la pandemia durante varios meses. Diversos estudios internacionales han reportado un impacto negativo sobre la enfermedad cardiovascular relacionado con las medidas sanitarias instituidas en distintos países ${ }^{(19,20)}$.

El Dr. Víctor Dayan y col. diseñaron un estudio ecológico, descriptivo, retrospectivo y analítico, con el objetivo primario de evaluar la incidencia de reperfusión de infartos en Uruguay desde el 13 de marzo al 30 de abril de 2020, y compararlo con los años anteriores para el mismo período. Como objetivos secundarios, los investigadores pretendieron identificar si hubo diferencias en los tiempos de reperfusión, mortalidad a 15 días luego del procedimiento, mortalidad global y cardio- vascular durante el primer semestre de 2020, en comparación con años anteriores ${ }^{(21)}$.

Para el desarrollo del estudio, los investigadores extrajeron los datos del Fondo Nacional de Recursos registrados en los años 2017 a 2020, e incluyeron a todos los pacientes que recibieron reperfusión por fibrinolíticos o ATC por IAMCEST en Uruguay. Los datos de mortalidad global y cardiovascular fueron extraidos a partir del informe del Ministerio de Salud Pública.

Los investigadores encontraron que, en el período estudiado en 2020, tanto la incidencia de IAMCEST como el número de pacientes tratados fue menor en comparación con los años 2017, 2018 y 2019, pero con similar tiempo de reperfusión y mortalidad a 15 días, y una menor mortalidad global en el 2020. No se detectaron diferencias en la mortalidad cardiovascular o debida a IAMCEST en este período.

Los autores concluyeron que la emergencia sanitaria se asoció a una disminución en la reperfusión de IAMCEST, y que esto se asoció a menor mortalidad global durante el primer semestre de 2020. Este trabajo fue galardonado como el mejor tema libre del Congreso.

\section{Correlación entre parámetros hemodinámicos y captación de ${ }^{18} \mathrm{Ffluoruro}$ mediante PET a corto plazo luego de sustitución valvular aórtica}

El reemplazo valvular es la primera línea de tratamiento en la estenosis aórtica severa sintomática. Existen dos tipos de válvulas cardíacas artificiales: las mecánicas, implantadas de forma quirúrgica, y las biológicas, que pueden ser implantadas de forma quirúrgica o percutánea. Este último abordaje ha crecido de manera exponencial en la última década. Aunque el uso de la bioprótesis ha aumentado, diversos grados de disfunción pueden aparecer a lo largo del tiempo ${ }^{(22)}$. Esta disfunción bioprótesica se define como la presencia de un gradiente medio > $20 \mathrm{mmHg}$, un área efectiva del orificio $<0,91,1 \mathrm{~cm}^{2}$, un índice de velocidad Doppler < 0,35 y/o insuficiencia protésica moderada-severa ${ }^{(23)}$, puede ser secundaria a deterioro estructural, deterioro no estructural, trombosis o endocarditis.

Recientemente se ha descrito que otros estudios de imagen, como el PET, podrían permitir detectar la disfunción de las bioprótesis de forma precoz. Basados en este postulado, la Dra. Amparo Fernández y col. seleccionaron un gru- po de 19 pacientes que fueron sometidos a sustitución valvular aórtica por bioprótesis porcina en dos centros nacionales, entre el 01/01/2019 y el 13/02/2020, con el objetivo de correlacionar los parámetros de deterioro estructural protésico demostrado mediante PET con ${ }^{18} \mathrm{~F}$-fluoruro con los parámetros hemodinámicos ecocardiográficos ${ }^{(24)}$.

Los pacientes seleccionados tuvieron una media de edad de 72 años, $42 \%$ de sexo masculino, $47 \%$ tenía lesiones coronarias, $42 \%$ tabaquista y 73\% presentaba dislipemia. Se realizó el PET a los 16,3 meses (15,9-16,9 meses) tras la sustitución valvular aórtica. Los autores encontraron una correlación negativa estadísticamente significativa entre la captación de ${ }^{18}$ Ffluoruro en el PET y el gradiente medio al año (Rho de Spearman, coeficiente de correlación -0,516, valor de $\mathrm{p}=0,028$ ), así como entre la captación y el gradiente máximo al año (Rho de Spearman, coeficiente de -0,589, valor de $\mathrm{p}=0,010$ ). Con estos resultados, los autores destacaron que la mayor captación de ${ }^{18} \mathrm{~F}$-Fluoruro en el PET se correlacionaba con menores gra- 
dientes medios y máximos al año, y remarcaron el rol emergente del PET para la detección precoz de la disfunción de la bioprótesis aórtica.

Luego de la presentación, el Tribunal Arbitral de Honor otorgó a la autora el premio a Mejor In- vestigador Joven del Congreso. Se extiende una felicitación especial a la Dra. Amparo Fernández por ser el segundo año consecutivo que es merecedora de este lauro.

\section{Indicación, resultados y mortalidad de la angioplastia coronaria con implante de stent en tronco de arteria coronaria izquierda no protegido}

En la actualidad, la lesión significativa del tronco de la coronaria izquierda (LSTCI) se diagnostica y se trata con más frecuencia, debido al avance de nuevas técnicas y tecnologías que permiten detectar y valorar de mejor forma este tipo de lesiones, como la ultrasonografía intravascular (IVUS), la medición de la fracción de flujo de reserva y la tomografía de coherencia óptica. Debido a esto, se han llevado a cabo estudios randomizados que demostraron que la ATC de LSTCI puede ser favorable en comparación a la cirugía de revascularización, en ciertos grupos de pacientes ${ }^{(25)}$.

En Uruguay, la disponibilidad de acceder a las nuevas tecnologías mencionadas es baja, y la evidencia del beneficio de ATC del LSTCI no está clara. La Dra. Jessica Russo y col. diseñaron un estudio retrospectivo y observacional, que incluyó 37 pacientes de un centro de Uruguay sometidos a ATC de LSTCI no protegido (sin cirugía de revascularización miocárdica previa), tanto de emergencia $(64,9 \%)$, dado por la presencia de ángor refractario o inestabilidad hemodinámica, como no emergencia o electiva $(35,1 \%)$. Se utilizó IVUS solo en 5 pacientes, sin cambios en la conducta terapéutica. La mortalidad global fue del 37,8\%, y el 16,2\% falleció intraprocedimiento. Tras un seguimiento con una mediana de 16,1 meses (IQ:
2,743,6 meses), la mortalidad en el primer mes fue del $21,6 \%$, mientras que en el resto del seguimiento fue del $20,7 \%$. La sobrevida media global fue de 51,6 $\pm 6,5$ meses. No se requirió una nueva revascularización en la evolución ${ }^{(26)}$.

Basados en estos hallazgos, los autores concluyen que la mayor indicación de ATC de LSTCI no protegido fue en situaciones de emergencia, la técnica de stent provisional fue la más usada en compromiso de bifurcación, la mortalidad se vinculó al contexto clínico y a la severidad anatómica, y el uso de IVUS no determinó modificaciones sustanciales en la conducta terapéutica. La expansión del estudio con una mayor población y una disponibilidad de tecnología más extensa para la valoración angiográfica podría aumentar el beneficio de la ATC de LSTCI no protegido, sobre todo en aquellos individuos que se encuentran en el grupo de ATC electiva.

El Consejo Editorial felicita a todos los autores por los trabajos presentados, cuya calidad metodológica enaltece a la SUC.

Carlos Guamán, ORCID 0000-0002-1065-1988

Keril Gianoni, ORCID 0000-0003-4168-4253

Estefanía de la Fuente, ORCID 0000-0003-1456-2250

David de Sosa, ORCID 0000-0003-0507-8723

\section{Bibliografía}

1. Campos-Quintero A, García-Montes J, CruzArias R, Zabal-Cerdeira C, Calderón-Colmenero J, Sandoval J. Endocardial pacing in infants and young children weighing less than 10 kilograms. Rev Esp Cardiol (Engl Ed) 2018; 71(1):4851. doi: 10.1016/j.rec.2017.02.036

2. McLeod K. Cardiac pacing in infants and children. Heart 2010; 96(18):1502-8. doi: 10.1136/ hrt.2009.173328

3. Gutiérrez-Larraya Aguado F, Zavanella Botta C. Estimulación cardiaca en pediatría. Cuadernos Técnicos 1999; 3:7-15. Disponible en: https://secardiologia.es/images/stories/secciones/estimulacion/ cuadernos-estimulacion/0399/estimulacion-cardia- ca-en-pediatria.pdf. [Consulta: 18 agosto 2021].

4. Ríos M, Segura G, Chiesa P, Cuesta A. Implante primario de marcapaso definitivo cardíaco en población pediátrica y evolución: experiencia de 15 años en centro de referencia cardiológico pediátrico en Uruguay. [Abstract]. Rev Urug Cardiol. 2021 May [Consulta: 15 Oct 2021];36 [aprox.1p.]. Disponible en: https://www.cardiosuc2021.suc.org.uy/temas_libres

5. Phillips K, Ansell J. Outpatient management of oral vitamin $\mathrm{K}$ antagonist therapy: defining and measuring high-quality management. Expert Rev Cardiovasc Ther 2008; 6(1):57-70. doi: 10.1586/14779072.6.1.57

6. Veeger N, Piersma-Wichers M, Tijssen J, Hi- 
llege $\mathbf{H}$, van der Meer J. Individual time within target range in patients treated with vitamin $\mathrm{K}$ antagonists: main determinant of quality of anticoagulation and predictor of clinical outcome. A retrospective study of 2300 consecutive patients with venous thromboembolism. Br J Haematol 2005; 128(4):513-9. doi: 10.1111/j.1365-2141.2004.05348.x

7. Marín A, Neira V, Aizman A, Paredes A, Palma S, Ruiz M, et al. Eficacia y seguridad del tratamiento anticoagulante oral con antagonistas de vitamina $\mathrm{K}$ en pacientes con prótesis valvulares cardíacas. Rev Chil Cardiol 2014; 33(1):27-32. doi: 10.4067/S0718-85602014000100003

8. Mirandetti E, Rebollo E, Gómez A, Florio L. Calidad de la anticoagulación oral con warfarina en una policlínica de cardiología, seguimiento de un año. [Abstract]. Rev Urug Cardiol. 2021 May [Consulta: 15 Oct 2021];36[aprox.1p.]. Disponible en: https://www.cardiosuc2021.suc.org.uy/temas_libres

9. Karkouti K, Wijeysundera D, Beattie W; Reducing Bleeding in Cardiac Surgery (RBC) Investigators. Risk associated with preoperative anemia in cardiac surgery: a multicenter cohort study. Circulation 2008; 117(4):478-84. doi: 10.1161/CIRCULATIONAHA.107.718353

10. Rössler J, Schoenrath F, Seifert B, Kaserer A, Spahn G, Falk V, et al. Iron deficiency is associated with higher mortality in patients undergoing cardiac surgery: a prospective study. Br J Anaesth 2020; 124(1):25-34. doi: 10.1016/j.bja.2019.09.016

11. Hernández M, Ramos C, Giménez D, Bazzino O, Cura L, Florio L. Valor pronóstico de la anemia leve/moderada y/o la ferropenia preoperatoria en el posoperatorio de cirugía cardiaca [Abstract]. Rev Urug Cardiol. 2021 May [Consulta: 15 Oct 2021];36 [aprox.1p.]. Disponible en: https://www. cardiosuc2021.suc.org.uy/temas_libres

12. Bassan R, Gibler W. Unidades de dolor torácico: estado actual del manejo de pacientes con dolor torácico en los servicios de urgencias. Rev Esp Cardiol 2001; 54(9):1103-9. doi: 10.1016/s03008932(01)76457-3

13. Farro I, Américo C, Martínez F, Lluberas N, Parma G, Aramburu J, et al. Impacto pronóstico del ecocardiograma de estrés con ejercicio físico negativo en una unidad de dolor torácico. Período 20192020 con seguimiento a un año [abstract]. Rev Urug Cardiol. 2021 May [Consulta: 29 Oct 2021];36 [aprox.1p.]. Disponible en: https://www.cardiosuc2021.suc.org.uy/sites/default/files/2021-04/ TL\%20Nro.\%2045.pdf.

14. Ibanez B, James S, Agewall S, Antunes M, Bucciarelli-Ducci C, Bueno H, et al; ESC Scientific Document Group. 2017 ESC Guidelines for the management of acute myocardial infarction in patients presenting with ST-segment elevation: The Task Force for the management of acute myocardial infarction in patients presenting with ST-segment elevation of the European Society of Cardiology (ESC). Eur Heart J 2018; 39(2):119-77. doi: 10.1093/ eurheartj/ehx393
15. Kelbæk H, Høfsten D, Køber L, Helqvist S, Kløvgaard L, Holmvang L, et al. Deferred versus conventional stent implantation in patients with ST-segment elevation myocardial infarction (DANAMI 3-DEFER): an open-label, randomised controlled trial. Lancet 2016; 387(10034):2199-206. doi: 10.1016/S0140-6736(16)30072-1

16. Albistur J, Loza G, Bachini J, Niggemeyer A, Falero N. Estrategia invasivo-conservadora en el infarto con elevación del ST. Prueba de concepto [Abstract]. Rev Urug Cardiol 2021 May [Consulta: 15 Oct 2021];36 [aprox.1p.]. Disponible en: https:// www.cardiosuc2021.suc.org.uy/temas_libres

17. Mackenzie JS, Smith DW. COVID-19: a novel zoonotic disease caused by a coronavirus from China: what we know and what we don't. Microbiol Aust 2020:MA20013. doi: 10.1071/MA20013

18. Andersen K, Rambaut A, Lipkin W, Holmes E, Garry R. The proximal origin of SARS-CoV-2. Nat Med 2020; 26(4):450-2. doi: 10.1038/s41591-0200820-9

19. Ball S, Banerjee A, Berry C, Boyle J, Bray B, Bradlow W, et al; CVD-COVID-UK Consortium. Monitoring indirect impact of COVID-19 pandemic on services for cardiovascular diseases in the UK. Heart 2020; 106(24):1890-7. doi: 10.1136/ heartjnl-2020-317870

20. Muhammad D, Abubakar I. COVID-19 lockdown may increase cardiovascular disease risk factors. Egypt Heart J 2021; 73(1):2. doi: 10.1186/s43044020-00127-4

21. Dayan V, Perna A, Soto E, Niggemeyer A, Piñeiro N, Fernández G, et al. Impacto cardiovascular de la pandemia por COVID-19 durante el primer semestre de 2020 en Uruguay. Estudio ecológico [Abstract]. Rev Urug Cardiol. 2021 May [Consulta: 15 Oct 2021];36 [aprox.1p.]. Disponible en: https://www.cardiosuc2021.suc.org.uy/temas_libres

22. Kostyunin A, Yuzhalin A, Rezvova M, Ovcharenko E, Glushkova T, Kutikhin A. Degeneration of bioprosthetic heart valves: update 2020. J Am Heart Assoc 2020; 9(19):e018506. doi: 10.1161/ JAHA.120.018506

23. Capodanno D, Petronio A, Prendergast B, Eltchaninoff H, Vahanian A, Modine T, et al. Standardized definitions of structural deterioration and valve failure in assessing long-term durability of transcatheter and surgical aortic bioprosthetic valves: a consensus statement from the European Association of Percutaneous Cardiovascular Interventions (EAPCI) endorsed by the European Society of Cardiology (ESC) and the European Association for Cardio-Thoracic Surgery (EACTS). Eur J Cardiothorac Surg 2017; 52(3):408-17. doi: 10.1093/ ejcts/ezx244

24. Fernández A, Loza G, Parma G, Florio L, Alonso $\mathbf{O}, \mathbf{N i e l l} \mathbf{N}$, et al. Correlación entre parámetros hemodinámicos y captación de 18F-Fluoruro mediante PET a corto plazo luego de sustitución valvular aórtica [Abstract]. Rev Urug Cardiol. 2021 May 
[Consulta: 15 Oct 2021]; 36 [aprox.1p.]. Disponible en: https://www.cardiosuc2021.suc.org.uy/temas_ libres

25. Bing R, Yong A, Lowe H. Percutaneous Transcatheter Assessment of the Left Main Coronary Artery: Current Status and Future Directions. JACC Cardiovasc Interv 2015; 8(12):1529-39. doi: 10.1016/j.jcin.2015.07.017
26. Russo J, Albistur J, Vignolo G. Indicación, resultados y mortalidad de la angioplastia coronaria con implante de stent en tronco de arteria coronaria izquierda no protegido [Abstract]. Rev Urug Cardiol. 2021 May [Consulta: 15 Oct 2021];36 [aprox.1p.]. Disponible en: https://www.cardiosuc2021.suc.org. uy/temas_libres

\section{Contribución de autores}

Carlos Guamán. Recolección de datos, redacción, correcciones y respuesta a editores.

Keril Gianoni. Recolección de datos, redacción.

Estefanía de la Fuente. Recolección de datos, redacción.

David de Sosa. Recolección de datos, redacción. 